\title{
X-ray and Neutron Emission Studies in a New Filippov Type Plasma Focus
}

\author{
A.R. Babazadeh ${ }^{1,2}$, M.V. Roshan ${ }^{2}$, H. Habibi², A. Nasiry², M. Memarzadeh ${ }^{2}$, \\ A. Banoushi ${ }^{1,2}$, M. Lamehi ${ }^{2}$, and S.M. Sadat Kiai ${ }^{1,2}$ \\ ${ }^{1}$ Technical University of Amirkabir, \\ Depatment of Physics, Tehran 15875 - 4413. \\ ${ }^{2}$ Nuclear Fusion Research Center, A.E.O.I, \\ P.O.Box 14155-1339, Tehran, Iran
}

Received on 26 June, 2001

\begin{abstract}
We have performed experimental comparative studies of the $\mathrm{x}$-ray and neutron emission generated by the new Filippov-type plasma focus "Dena", (90 kJ, $25 \mathrm{kV}, 288 \mu \mathrm{F})$ in the pressure range of 0.6-1 torr. Time-integrated and time-resolved detectors, together with an x-ray pin-hole camera, along with a Be filter of $10 \mu \mathrm{m}$ thickness have been used. For a working gas of neon and a flat insert anode, the maximum soft and hard x-rays (SXR-HXR) yield obtained was $16 \mathrm{~V}$ and $1.5 \mathrm{~V} /$ shot over a $4 \pi$ solid angle, respectively, for a charging voltage range of $16-20 \mathrm{kV}$. As for the argon gas, the similar results such as 3.5 and $2 \mathrm{~V} /$ shot have been found, leading to a total conversion efficiency of x-ray emission of $0.09 \%$ (for neon) and $0.03 \%$ (for argon) of the stored energy. These efficiencies have been improved by the employment of a conic insert anode up to $0.4 \%$ and $0.1 \%$. With deuterium puffing gas and a flat insert anode, the maximum emission yield has been found to be $2.5 \mathrm{~V}$ for SXR and $1 \mathrm{~V}$ for HXR/shot which produce an ultimate emission profile width (FWHM) of 70-90 $\mathrm{ns}$ for x-rays and neutrons, giving rise to a maximum neutron yield of $1.2 \times 10^{9}$. Nevertheless, the maximum yield has been increased up to 5.5 times with the conic insert anode. In order to increase the neutron yield, we have introduced a krypton admixture to the deuterium filling gas and found that, for a krypton pressure of about 0.1 torr, the neutron yield increases by a factor of 3.5 for the flat insert and 1.5 for the conic insert anodes.
\end{abstract}

\section{Introduction}

Since its development in the 1960's, the plasma focus discharge is recognized as a powerful and excellent device in the production of three principal kinds of radiation, such as fast neutrons proceeding from deuterium nuclear fusion reactions, intense $\mathrm{x}$ rays, coming from different filling gases and the anodes in the discharge chamber and, energetic charged particles such as ions and electrons.

Regarding the need to improve the characteristics of these radiations, in recent years there has been considerable effort in developing and enhancing the basic mechanisms of the phenomena related to the plasma focus facilities. For this purpose, there are many independent laboratory works published in the literature, some of which we will be considered here.

In 2000, Castillo et al. [1] investigated the possible coexistence of thermal and non thermal mechanisms through measurement of the neutron yield, in comparison with time- resolved intensity measurements of the soft and hard x-ray radiations. The paper concluded that there is a coexistence of both mechanisms competing for the neutron yield in their plasma focus device.

Bostik et al. [2] considered the correlations between ion beam intensity, which is dependent on the discharge gas pressure, and neutron yield, and hard x-ray intensity. They found that the maximum radiation of ion beam and the related neutron yield increase with the hard x-ray intensity.

The x-ray emission from plasma focus devices was reported by Harries et al. [3]. They stated that x-rays with low and high energy originate both in the plasma and on the anode surface, respectively.

Herold et al. [4] worked on the geometry and structure of the inner and outer electrodes, the material and configuration of the insulator. Their paper points out that the sputtered material from the electrode that gets into the current sheath, produces plasma impurity contamination and consequently results in a deterioration of the neutron yield from the plasma focus.

The deterioration of the neutron yield caused by 
the anodes erosion was investigated by Routel et al. [5]. They showed that the neutron yield obtained when using a tungsten- copper alloy anode was almost twice as much as for an aluminum anode.

Zakaullah et al. [6] employed different anode shapes with argon filling gas and reported that the radiation yield and the gas pressure needed for good focusing are found to be strongly dependent on the anode shape.

Several research works have been devoted to investigate the effect of gas admixture on the behavior of the focused plasma [7-10]. More recently, Babazadeh et al. [11] worked on an experimental study of x-ray radiation yield in a new Filippov-type plasma focus $(25 \mathrm{kV}, 90$ $\mathrm{kJ}$ ) operated with low pressure neon and neon-krypton admixture gases. The result of their investigation was that the krypton admixture gas generally causes the soft $\mathrm{x}$-ray yield to decrease down to zero, whereas the hard $\mathrm{x}$-ray emission yield attains a maximum value at the optimum pressure of krypton gas.

This work seeks to find out if the plasma radiation intensity varies with anode shape and the type of gases used. In this connection, one of the objectives is to measure the intensity of the soft and hard x-ray together, as well as the neutron yield.

In this article we focus our attention on the use of two types of insert anodes, i.e. flat or conic, with different given gases to evaluate a comparative study of the radiated intensities. The effect on radiation of using a krypton admixture in the deuterium filling gas is also considered.

\section{Experimental setup}

The experiments have been performed in the new Filippov type "DENA" plasma focus system, with a so called flat geometry of electrodes, which provides an opportunity to vary the operating discharge conditions on a large scale (for Filippov type plasma focus see for example [12-13]). The discharge is powered by a $288 \mu \mathrm{F}$ capacitor bank, at operating voltages up to $25 \mathrm{kV}$ (90 $\mathrm{kJ}$ ), giving a peak discharge current of about 2.8 MA. Fig. 1 shows the electrodes assembly of the plasma focus system. The spark gap is used at a pressure of about $1.2 \times 10^{-2}$ torr, to transfer the charge through the transmission lines of $18 \mathrm{nH}$ inductance, to the focus anode, This spark gap has a low inductance $\leq 0.2$ $\mathrm{nH}$ which we ignore, as compared to the total inductance of the system; $23 \mathrm{nH}$ including $2 \mathrm{nH}$ from the capacitor bank and $3 \mathrm{nH}$ from the discharge chamber inductance. The discharge current rise time is about $2.8 \mu \mathrm{s}$, with an effective impedance of $9 \times 10^{-3} \mathrm{Ohm}$.
The anode is placed at the center of the vacuum chamber. It is a disc of $50 \mathrm{~cm}$ diameter and $15.5 \mathrm{~cm}$ high, made of copper, and at its center, there is a conic insert anode made of copper-tungsten alloy, that can be easily replaced. The anode is surrounded by a cathode made of stainless steel of $71.5 \mathrm{~cm}$ inner diameter, insulated from the anode by porcelain with a height of $12 \mathrm{~cm}$, and a diameter of $48 \mathrm{~cm}$.

The discharge current and its derivative are measured by a Rogowski coil with 1115 turns and a coil with 20 turns, respectively. A magnetic probe, located on the side of the cathode ring monitors the current sheath structure during the rundown phase of the plasma discharge. The time-resolved soft x-ray emission is measured by a PIN diode (SPPD type) with a $10 \mu \mathrm{m}$ thick beryllium filter, in order to minimize the effect of visible light of the plasma. This detector is located on a sideon position at $90^{\circ}$ from the axis. The time-integrated soft x-ray images of the plasma column were obtained with a pinhole camera with a small 10-100 $\mu$ m aperture. The time-integrated measurement of hard x-ray intensity was registered by a NaI- scintillator coupled to a photo element coaxial (PhEC) detector placed on top of the device. The time-resolved hard x-ray and neutron emission was measured by a photomultiplier (PM-53 type) coupled to a NE-102 plastic scintillator with a $5 \mathrm{~ns}$ resolution. A Geiger-Muller counter covered by an indium foil, with $0.23 \mathrm{~mm}$ thickness placed in a polyethylene moderator; measures the number of neutrons. We have also used time-integrated thermoluminescent (TLD) dosimeters, made of LiF, to monitor the hard x-ray emissions.

A four-channel computer with executing data acquisition system together with two GPIB oscilloscopes, with a maximum resolution of 5 ns for each channel are responsible for signal acquisition.

\section{Results and discussion}

For the purpose of a comparative study, we consider first, the x-ray emission for the two insert anode configurations with neon and argon filling gases. For the discharges, the working gas pressure, including deuterium, ranges from 0.6 to 1.0 torr, using charging voltages between 16 to $20 \mathrm{kV}$. For each selected voltage, we have registered the optimum gas pressure related to the maximum x-ray emissions and used them in the experiments. In order to reduce the effect of plasma impurities contamination, the chamber was pumped out and filled with fresh gas every ten shots. 


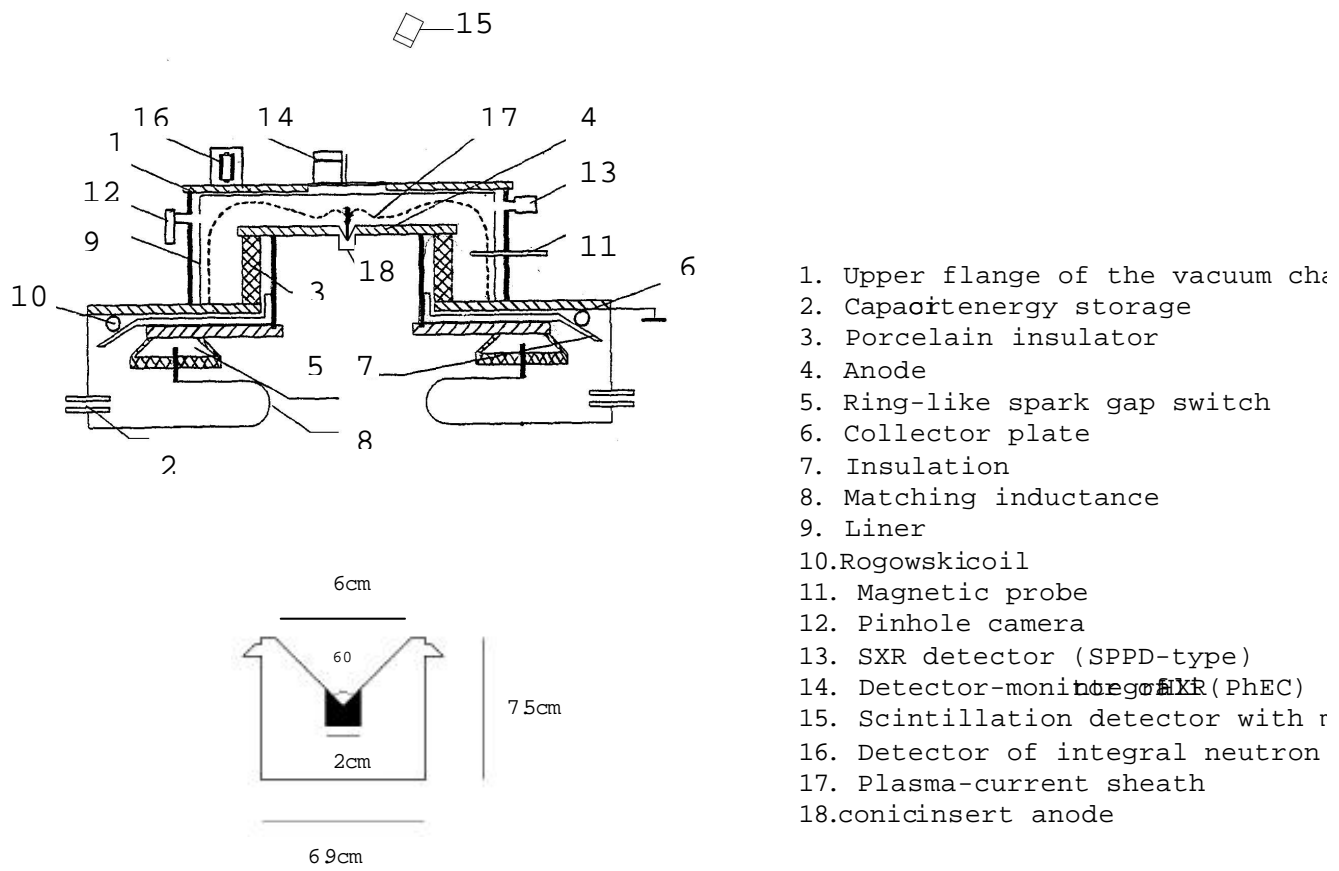

Conic insert anode

Figure 1. A schematic diagram showing the plasma focus electrodes and diagnostics.
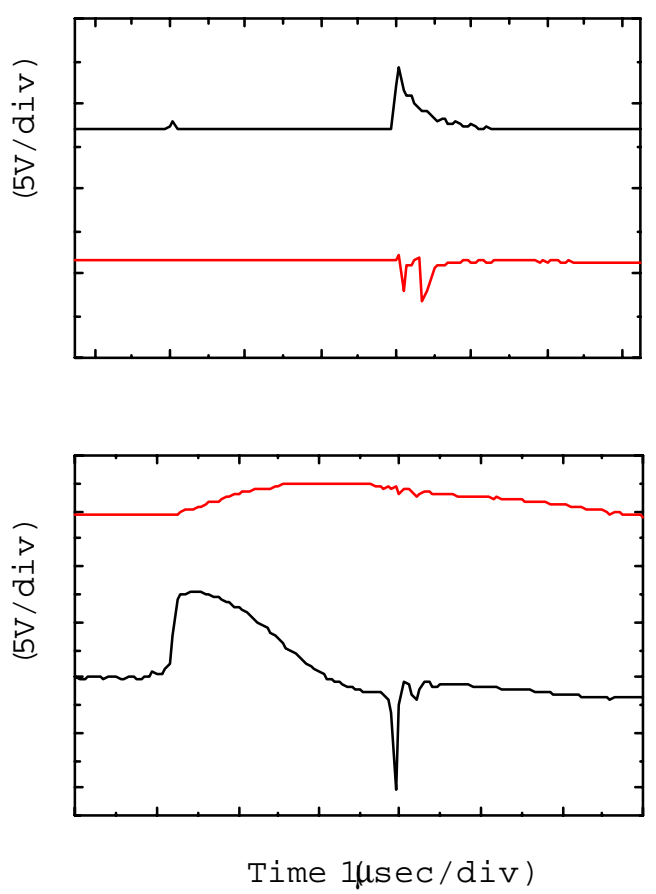

Figure 2. A typical oscilloscope signal using deuterium filing gas with a flat anode showing four simultaneous measurements (from top to bottom): soft x-ray pulse, hard x-ray and neutron pulses, total discharge current and the total current derivative.

Fig. 2 shows typical oscillograms of the signals obtained from deuterium filling gas. This figure displays signals of the soft $\mathrm{x}$-ray, hard $\mathrm{x}$-ray and neutron emissions from the PIN diode and scintillatorphotomultiplier detector, respectively, the total discharge current from the Rogowski coil, and the current derivative (dI/dt). From the current derivative signal, the existence of zones with sufficient width for time measurements of the focus pinch duration can be noted. It is generally accepted that the negative peak of current derivative time, corresponds to the minimum pinch diameter [14]. From consideration of the corresponding streak photograph of a pinch (see for example [15]), it is probably possible to associate two time zones in the $\mathrm{dI} / \mathrm{dt}$ signal. The first time zone corresponds to the beginning of the pinch, when $\mathrm{dI} / \mathrm{dt}$ is maximum, until $\mathrm{dI} / \mathrm{dt}=0$. In this region the diameter of the pinch is fairly constant. The second time zone is from $\mathrm{dI} / \mathrm{dt}=$ 0 until the appearance of the spike in the discharge current. From this time on, a burst in the pinch, causes a sudden increase in its diameter.

We have displayed in Fig.3 the experimental measurements of the soft and hard x-ray intensities against the different discharge voltages. In this experiment flat and conic insert anodes were used with neon and argon filling gases. Fig.3, presents (a) the soft and (b) the hard $\mathrm{x}$-ray intensities, respectively. As can be seen, a 
significant increase in the values of the soft $\mathrm{x}$-ray intensities is observed from both gases with the conic insert anode. We have obtained maximum values of soft and hard x-ray yields about $16 \mathrm{~V}$ and $1.5 \mathrm{~V} /$ shot in $4 \pi$ geometry, respectively, with the flat insert anode and neon working gas. For argon filling gas these values are $3.5 \mathrm{~V}$ and $2 \mathrm{~V} /$ shot, respectively. The results obtained indicate a total conversion efficiency of $\mathrm{x}$-ray yield of about $0.09 \%$ (for neon at $20 \mathrm{kV}$ ) and $0.03 \%$ (for argon at $18 \mathrm{kV}$ ) of the discharge stored energy. These efficiencies have been improved by the employment of the conic insert anode up to $0.4 \%$ (for neon) and 0.1 $\%$ (for argon).

The present investigation confirms the important fact that the shape and the material of the anode has significant effect on the production of x-ray emissions. However, with the neon and argon gases we have found a maximum increment factor of about 5 for the soft x-ray intensity with conic anode, as compared to the results using the flat insert anode. The equivalent comparison for the hard $\mathrm{x}$-rays is 1.5 .
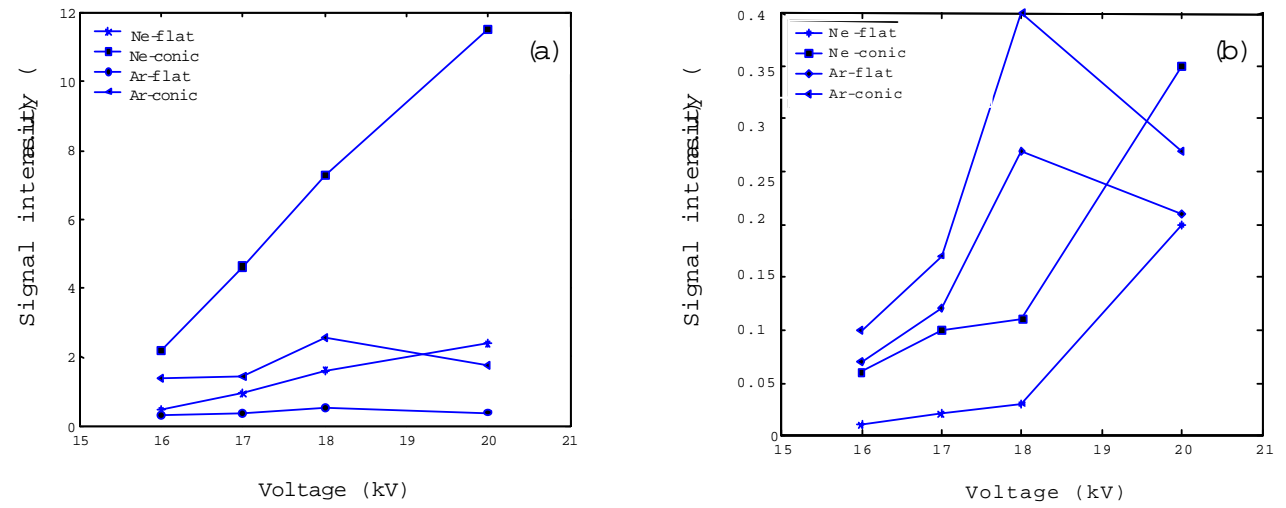

Figure 3. (a) Soft X-ray and (b) hard X-ray signal intensities against the discharge voltages from neon and argon filling gases with the two anode configurations.

Fig. 4 shows two soft $x$-ray pin-hole images of the focus plasma for neon filling gas, but different insert anodes; (a) flat anode, and (b) conic insert anode. Fig. 4(b) clearly indicates a wider emitting region due to a better pinch of the focus plasma.

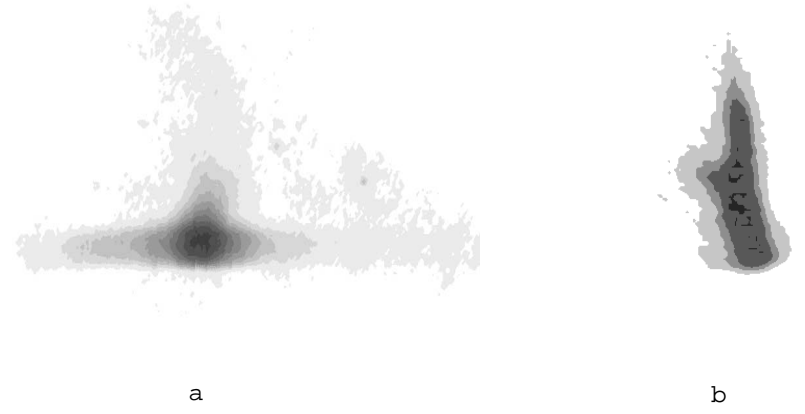

Figure 4. Time integrated pinhole images of soft X-ray emission from neon focus with (a) flat, and (b) conic anodes.

In more recent work, we have studied the behavior of the neutron yield through an experiment similar to the previous one, except in one case, we have examined the effect of krypton admixture gas on the neutron and $\mathrm{x}$-ray emissions. In this study, first we have used only deuterium puffing gas and a flat insert anode to obtain the soft and hard $\mathrm{x}$-ray intensities. We have found maximum values of $2.5 \mathrm{~V} /$ shot for soft $\mathrm{x}$-rays and $1 \mathrm{~V} /$ shot for hard x-rays over a $4 \pi$ solid angle, and a measured maximum neutron yield of $1.2 \times 10^{9}$.

The neutron yield was measured using a GeigerMuller counter covered by an indium foil with $0.23 \mathrm{~mm}$ thickness which was calibrated by a Cf standard neutron source. The neutron yield enhancement has been improved by a factor of 5.5 using a conic insert anode. However, an ultimate emission profile width (FWHM) of 70-90 ns was measured for the x-ray and neutron emissions. Fig. 2 shows the neutron pulse appeares 50 ns after the hard x-ray signal, as measured by the plastic scintillator-photomultiplier detector. This delay time corresponds to the time of flight of $2.45 \mathrm{MeV}$ neutrons from the outgoing discharge to the scintillator. The effect of gas admixture on the intensities of the soft x-ray, hard x-ray and neutron emissions was studied by adding 0.1 torr of krypton gas. The results obtained with the flat insert anode are presented in Fig. 5. The intensities of soft and hard x-rays were increased with the krypton admixture, nevertheless, the hard $\mathrm{x}-$ ray intensity increment is more vigorous. Furthermore, the maximum increment factors found are about 5.5 
for hard x-ray and 1.5 for soft x-ray intensities. Fig. 6 presents the soft $\mathrm{x}$-ray pin-hole images; (a) only deuterium, and (b) deuterium krypton admixture.

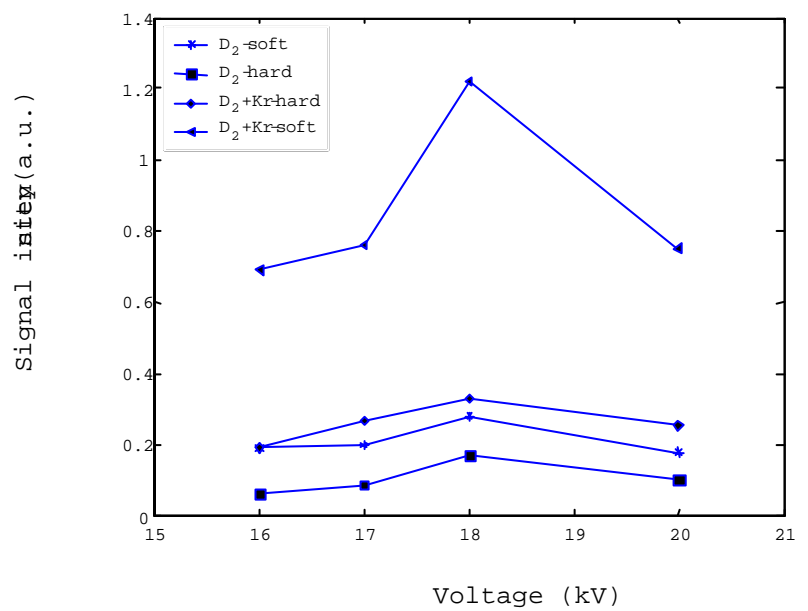

Figure 5. Soft and hard X-ray signal intensities against discharge voltages from deuterium and deuterium-krypton admixture with flat anode.

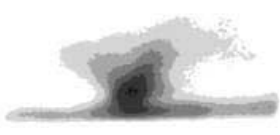

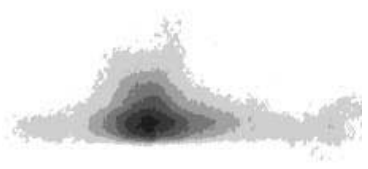

b
Figure 6. Time integrated pinhole images of soft X-ray emission from (a) deuterium (b) deuterium-krypton admixture.

Finally, regarding the neutron yield, the effect of the krypton admixture gas produced an increase factor of 3.5 (flat anode) and 1.5 (conic anode). Fig. 7 shows the neutron yield versus discharge voltages corresponding to the deuterium and deuterium- krypton with two anode configurations.

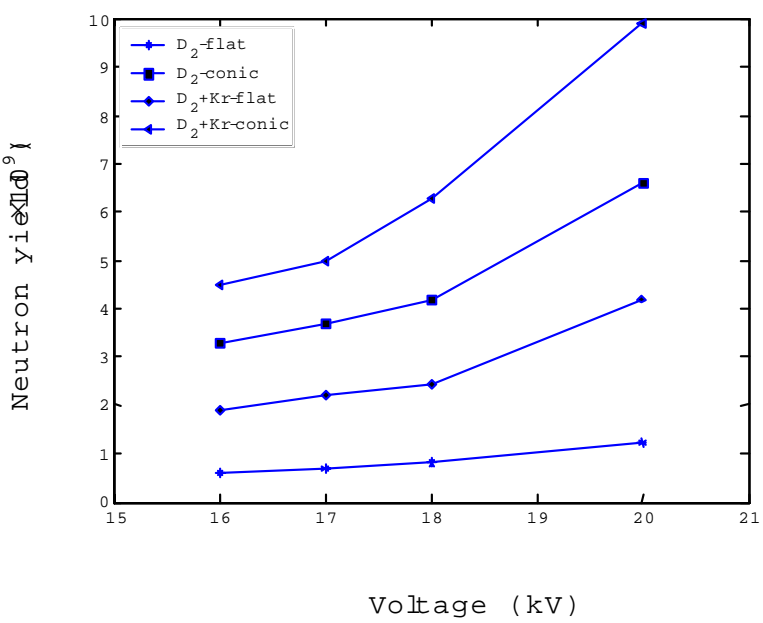

Figure 7. Neutron emission versus the discharge voltages from deuterium and deuterium-krypton admixture with the two anode configurations.

\section{Conclusions}

Comprehensive plasma emissions by means of different anode shapes, working gases, gas admixtures and discharge voltages, which have been discussed in recent years, could be easily investigated experimentally in this work on the behavior of the focus plasma. With the help of soft and hard x-ray and the neutron emissions from neon, argon and deuterium filling gases, one is able to obtain some important information about the plasma radiation yield phenomena.

Studies of the soft $\mathrm{x}$-rays from these working gases, with the employment of a conic anode in a new Filippov-type plasma focus device, have revealed the fact that the signal intensities show higher yield performances (with respect to the flat anode), compared to the hard x-ray intensities.

The addition of krypton admixture to the deuterium working gas, in the case of a flat insert anode, also increased the maximum yield of the soft and hard $\mathrm{x}-$ ray emissions. Nevertheless, this increment is much higher for the hard x-ray signals. However, we could not achieve this maximum yield for the conic insert anode, because the deuterium pressure was low (less than 1 torr), this might be the subject of future study.

The effect of krypton admixture to the deuterium filling gas has also enhanced the neutron yield by a factor of about 3.5 for the case of a flat anode and 1.5 for a conic insert anode.

\section{References}

[1] F. Castillo, M. Milanese, R. Moroso and J. Pouzo, J. Phys. D: Appl. Phys. 33, 141 (2000).

[2] W. H. Bostick, H. Kilic, V. Nardi and C. W. Powell, Nucl. Fusion 33, 413 (1993).

[3] W. L. Harries, J. H. Lee and D. R. McFarland, Plasma Phys. 20, 95 (1978).

[4] H. Herold, A. Jerzykkiewcz, M. Sadowski and H. Schmidt, Nucl. Fusion 29, 1255 (1989).

[5] R. K. Rout, A. Shyam and V. Chitra, Ann. Nucl. Energy 18, 357 (1991).

[6] M. Zakaullah, A. Omar Imtiaz Ahmad, G. Mortaza and M. M. Beg, Plasma Sources Sci. Technol. 5, 544 (1996).

[7] J. S. Brzosko and V. Nardi, Phys. Lett. A 155, 162 (1991).

[8] J. S. Brzosko, N. Nardi, J. R. Brzosko and Goldstein, Phys. Lett. A192, 250 (1991).

[9] M. Favre, P. Silvia, P. Choi, H. Chuaqui, C. Dumitrescu-Zoita and E. S. Wyndham, IEEE Trans. On Plasma Sci. 26, 1154 (1998). 
[10] L. Jakubowski, M. Sadowski, J. Zebrowski and E. Baronova, SINS Annual Report (1995) p. 96.

[11] A. R. Babazadeh, H. Habibi, A. Nasiry, M. V. Roshan and S. M. Sadat kiai, accepted paper for publication in the proceedings of the International Symposium PLASMA-2001, 19-21 September, 2001, Warsaw, Poland.

[12] N. V. Filippov, Review of Experimental Works on Study of Plasma Focus performed in Kurchatov In- stitute, Fizika Plazmy (Sov. J. Plasma Phys.) 9, 24 (1983).

[13] N. V. Filippov, T. I. Filippova, M. A. Karakin, et. al., Fillipov Type Plasma Focus as Intense Source of Hard X- rays, IEEE Trans. Plasma Science 24, 1215 (1996).

[14] F. M. Castillo-Mejia, M. Milanese, R. Moroso and J. Pouzo, J. Phys. D: Appl. Phys. 30, 1499 (1997).

[15] J. D. Sethian, A. E. Robson, K. A. Gerber, and A. W. Desilva, Physical Review Letters 59, 892 (1987). 